\title{
Overview of TJ-II experiments
}

\author{
J. Sánchez ${ }^{1}$, M. Acedo ${ }^{1}$, A. Alonso ${ }^{1}$, J. Alonso ${ }^{1}$, P. Alvarez ${ }^{1}$, F. de Aragón ${ }^{1}$, E. Ascasíbar ${ }^{1}$, \\ A. Baciero $^{1}$, R. Balbín ${ }^{1}$, L. Barrera ${ }^{1}$, E. Blanco ${ }^{1}$, J. Botija $^{1}$, B. Brañas ${ }^{1}$, E. de la Cal ${ }^{1}$, \\ E. Calderón ${ }^{1}$, I. Calvo ${ }^{1}$, A. Cappa ${ }^{1}$, J.A. Carmona ${ }^{1}$, B.A. Carreras ${ }^{2}$, R. Carrasco ${ }^{1}$, \\ F. Castejón ${ }^{1}$, G. Catalán ${ }^{1}$, A.A. Chmyga ${ }^{3}$, N.B. Dreval ${ }^{3}$, M. Chamorro ${ }^{1}$, S. Eguilior ${ }^{1}$, \\ J. Encabo ${ }^{1}$, L. Eliseev ${ }^{4}$, T. Estrada ${ }^{1}$, A. Fernández ${ }^{1}$, R. Fernández ${ }^{1}$, J.A. Ferreira ${ }^{1}$, \\ J.M. Fontdecaba ${ }^{1}$, C. Fuentes ${ }^{1}$, J. de la Gama ${ }^{1}$, A. García ${ }^{1}$, L. García ${ }^{5}$, I. García-Cortés ${ }^{1}$, \\ J.M. García-Regaña ${ }^{1}$, B. Gonçalves ${ }^{6}$, J. Guasp ${ }^{1}$, J. Herranz ${ }^{1}$, A. Hidalgo ${ }^{1}$, C. Hidalgo ${ }^{1}$, \\ R. Jiménez-Gómez ${ }^{1}$, J.A. Jiménez ${ }^{1}$, D. Jiménez ${ }^{1}$, I. Kirpitchev ${ }^{1}$, A.D. Komarov ${ }^{3}$, \\ A.S. Kozachok ${ }^{3}$, L. Krupnik ${ }^{3}$, F. Lapayese ${ }^{1}$, M. Liniers ${ }^{1}$, D. López-Bruna ${ }^{1}$, \\ A. López-Fraguas ${ }^{1}$, J. López-Rázola ${ }^{1}$, A. López-Sánchez ${ }^{1}$, E. de la Luna ${ }^{1}$, G. Marcon ${ }^{1}$, \\ F. Martín ${ }^{1}$, L. Martínez-Fresno ${ }^{1}$, K.J. McCarthy ${ }^{1}$, F. Medina ${ }^{1}$, M. Medrano ${ }^{1}$, \\ A.V. Melnikov ${ }^{3}$, P. Méndez ${ }^{1}$, E. Mirones ${ }^{1}$, B. van Milligen ${ }^{1}$, I.S. Nedzelskiy ${ }^{6}$, \\ M. Ochando ${ }^{1}$, J. Olivares ${ }^{1}$, R. Orozco ${ }^{1}$, P. Ortiz ${ }^{1}$, J.L. de Pablos ${ }^{1}$, L. Pacios ${ }^{1}$, I. Pastor ${ }^{1}$, \\ M.A. Pedrosa ${ }^{1}$, A. de la Peña ${ }^{1}$, A. Pereira ${ }^{1}$, D. Pérez-Risco ${ }^{1}$, A. Petrov ${ }^{4}$, S. Petrov ${ }^{7}$, \\ A. Portas ${ }^{1}$, D. Rapisarda ${ }^{1}$, L. Ríos ${ }^{1}$, C. Rodríguez ${ }^{1}$, L. Rodríguez-Rodrigo ${ }^{1}$, \\ E. Rodríguez-Solano ${ }^{1}$, J. Romero ${ }^{1}$, A. Ros $^{1}$, A. Salas ${ }^{1}$, E. Sánchez ${ }^{1}$, M. Sánchez ${ }^{1}$, \\ E. Sánchez-Sarabia ${ }^{1}$, X. Sarasola ${ }^{1}$, K. Sarksian ${ }^{8}$, C. Silva ${ }^{6}$, S. Schchepetov ${ }^{8}$, \\ N. Skvortsova ${ }^{8}$, A. Soleto ${ }^{1}$, F. Tabarés ${ }^{1}$, D. Tafalla ${ }^{1}$, J. Tera ${ }^{1}$, A. Tolkachev ${ }^{1}$, V. Tribaldos ${ }^{1}$, \\ V.I. Vargas ${ }^{1}$, J. Vega ${ }^{1}$, G. Velasco ${ }^{1}$, M. Weber ${ }^{1}$, G. Wolfers ${ }^{1}$, S.J. Zweben ${ }^{9}$ and B. Zurro ${ }^{1}$

\footnotetext{
${ }^{1}$ Laboratorio Nacional de Fusión. EURATOM-CIEMAT, Madrid, Spain

2 Oak Ridge National Laboratory, Oak Ridge, USA

${ }^{3}$ Institute of Plasma Physics, NSC KIPT, Kharkov, Ukraine

${ }^{4}$ Institute of Nuclear Fusion, RNC Kurchatov Institute, Moscow, Russia

${ }^{5}$ Universidad Carlos III, Madrid, Spain

${ }^{6}$ Associação EURATOM/IST, Centro de Fusão Nuclear, Lisboa, Portugal

${ }^{7}$ A.F. Ioffe Physical Technical Institute, St Petersburg, Russia

${ }^{8}$ General Physics Institute, Russian Academy of Sciences, Moscow, Russia

${ }^{9}$ Princeton Plasma Physics Laboratory, Princeton, NJ, USA

E-mail: Joaquin.sanchez@ciemat.es
}

Received 22 December 2006, accepted for publication 30 April 2007

Published 19 September 2007

Online at stacks.iop.org/NF/47/S677

\begin{abstract}
This paper presents an overview of experimental results and progress made in investigating the link between magnetic topology, electric fields and transport in the TJ-II stellarator. The smooth change from positive to negative electric field observed in the core region as the density is raised is correlated with global and local transport data. A statistical description of transport is emerging as a new way to describe the coupling between profiles, plasma flows and turbulence. TJ-II experiments show that the location of rational surfaces inside the plasma can, in some circumstances, provide a trigger for the development of core transitions, providing a critical test for the various models that have been proposed to explain the appearance of transport barriers in relation to magnetic topology. In the plasma core, perpendicular rotation is strongly coupled to plasma density, showing a reversal consistent with neoclassical expectations. In contrast, spontaneous sheared flows in the plasma edge appear to be coupled strongly to plasma turbulence, consistent with the expectation for turbulent driven flows. The local injection of hydrocarbons through a mobile limiter and the erosion produced by plasmas with well-known edge parameters opens the possibility of performing carbon transport studies, relevant for understanding co-deposit formation in fusion devices.
\end{abstract}

PACS numbers: 52.55.Dy, 52.55.Hc, 52.70.-m

(Some figures in this article are in colour only in the electronic version) 


\section{Introduction}

The magnetic topology is an important ingredient of plasma confinement in magnetic traps. Plasma flows also play a crucial role in transport in magnetically confined plasmas, and clarifying the contribution of neoclassical effects and turbulence to flows is a key outstanding issue. Due to the flexibility of stellarator devices, such as TJ-II, these are ideal laboratories to study the relation between magnetic topology, electric fields, flows and transport. The results presented in this paper were obtained in plasmas created and heated by electron cyclotron resonance heating $(\mathrm{ECRH})(2 \times 300 \mathrm{~kW}$ gyrotrons, at $53.2 \mathrm{GHz}$, 2nd harmonic, X-mode polarization) and neutral beam injection (NBI). The ECRH was coupled to the plasma via two quasi-optical transmission lines, placed at stellarator-symmetric positions, equipped with an internal steered mirror. Beams of $400 \mathrm{~kW}$ port-through $\left(\mathrm{H}_{0}\right)$ power at $30 \mathrm{kV}$ were injected into target plasmas created using one or both ECRH lines. Recent improvements in plasma diagnostics have led to a better understanding of the confinement properties of TJ-II, including a test of the collisional-radiative model by a supersonic He beam [1], the development of a dedicated NBI system and charge-exchange diagnostic [2], a twocolour infrared interferometer [3] and spectroscopy [4]. The paper is organized as follows: in section 2, properties of transport and electric fields in ECRH and NBI plasmas are discussed. In section 3, the link between core transitions and magnetic topology is studied. Plasma rotation and momentum re-distribution mechanisms are discussed in section 4. MHD studies are reported in section 5. Plasma-wall studies are presented in section 6. Finally, conclusions are presented in section 7 .

\section{Confinement and electric fields in ECRH and NBI plasmas}

Global and local transport studies. The global energy confinement time of TJ-II follows the new ISS04 scaling [5], which is based on data from nine major stellarators, and shows robust dependences on density and heating power. Dependences on other operational parameters, i.e. the major and the minor radii, magnetic field and the rotational transform, have been included based on inter-machine analysis. It turns out that there is a systematic offset of energy confinement between magnetic configurations, and its size correlates with the effective helical ripple of the external helical field.

A local analysis of electron heat diffusion, assuming pure diffusion and negligible convective heat fluxes, has been also performed. A density scan indicated that there is no significant change in $\chi_{\mathrm{e}}$ with density inside $\rho=0.4$ in the studied $n_{\mathrm{e}}$ range $\left(0.4 \leqslant\left\langle n_{\mathrm{e}}\right\rangle \leqslant 1.0 \times 10^{19} \mathrm{~m}^{-3}\right)$, whereas in the region $0.5<\rho<0.8, \chi_{\mathrm{e}}$ decreased with density [6]. The observed systematic dependences of plasma potential and density (see the next paragraph) indicate that, at least in the density gradient zone of these plasmas, the radial electric field and the heat confinement are closely linked magnitudes. A rotational transform scan showed an overall decreasing trend of $\chi_{\mathrm{e}}$ with iota.

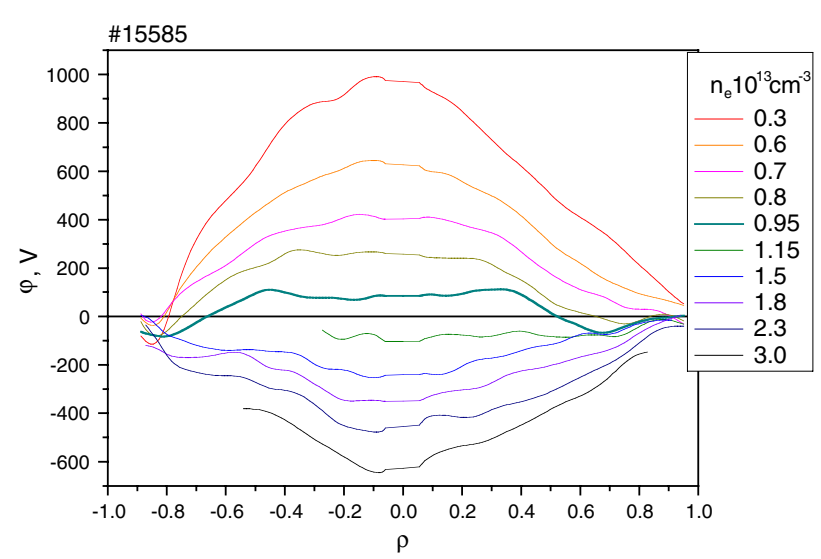

Figure 1. Plasma potential profiles as density increases from the pure ECRH phase to the ECRH+NBI one (from top (red) to bottom (grey)).

Electric fields in ECRH and NBI plasmas. The direct measurement of the electric potential and its fluctuations in the core plasma is of primary importance to understand the mechanisms of confinement improvement and the role of the electric field in plasma confinement. A heavy ion beam probe diagnostic $\left(\mathrm{Cs}^{+}\right.$with energies up to $\left.125 \mathrm{keV}\right)$ was used to study the plasma electric potential directly, with a good spatial $(\sim 1 \mathrm{~cm})$ and temporal $(\sim 2 \mu \mathrm{s})$ resolution.

It is well known that the plasma potential in stellarator devices (and the electric field) changes from positive to negative values as the density is increased [7]. Recent TJ-II experiments have investigated (shot by shot in stationary plasmas, as well as dynamically) the evolution of plasma potential profiles versus plasma density in a systematic way. Figure 1 shows the variation of the plasma potential as the density rises during an $\mathrm{ECH}+\mathrm{NBI}$ discharge. The smooth change from a positive to a negative plasma potential in the core plasma region is now known to be a systematic feature, not dependent on the TJ-II configuration.

Kinetic studies. The electron distribution function has been investigated in different confinement regimes, as well as changes in direct losses. The intensity and spatial distribution of the direct losses showed a strong dependence on plasma parameters [8]. Figure 2 shows direct losses versus plasma density, in a discharge with transitions between two confinement regimes, with high (black dots) and low (green circles) convective losses. The observed direct losses decrease with increasing plasma density and also depend on plasma confinement regimes. In addition, the response of convective particle losses to induced fast changes in the radial electric field was studied, through their effect on the total plasma radiation. The observed up-down and in-out radiation changes during electrode biasing and ECRH power switch on-off experiments (see figure 3 [9]) are consistent with calculated electric fieldinduced particle drift modifications [10].

Next, the effect of suprathermal electrons on impurity ionization states has been investigated, using iron impurities injected using the laser ablation technique. Recent experimental observations have shown evidence of a shift to higher charge states of iron when non-Maxwellian electron distributions are present. This phenomenon 

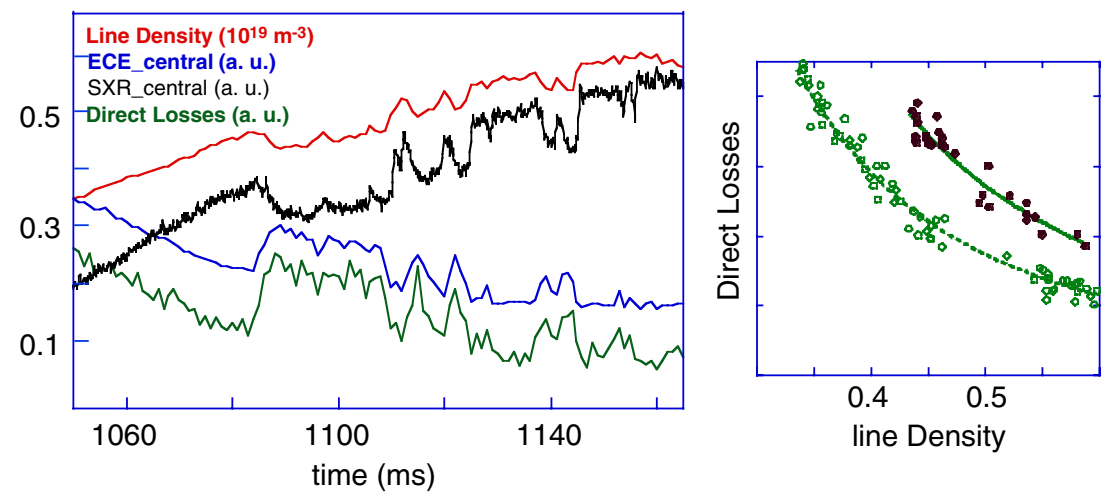

Figure 2. (Left) Time evolution of suprathermal electron losses to the inner chamber wall, as monitored with a soft $\mathrm{x}$-ray detector (33 $\mu \mathrm{m}$ Be foil), chord integrated soft x-ray emission ( $8 \mu \mathrm{m}$ Be foil), line-averaged electron density and central ECE signal. (Right) Dependence of the direct loss intensity with average density for high and low convective loss regimes.
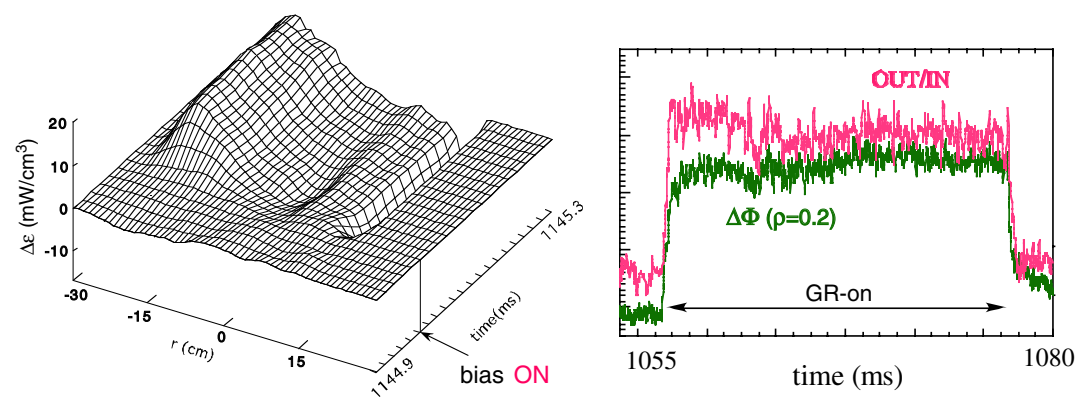

Figure 3. (Left) Temporal evolution of the radial profiles (along the horizontal plasma axis) of the emissivity perturbation induced by electrode biasing. (Right) Radiation asymmetry induced by ECRH modulation and simultaneous change in the central plasma potential.

could help understand why modelling of impurity transport coefficients, using theoretical ionization and recombination rates obtained by averaging over Maxwellian electron distributions, need adjustable anomalous diffusion and convective velocity coefficients to correctly reproduce the experiments [11].

Statistical description of transport. The improvement in plasma modelling tools allows a better study of the complex coupling between profiles, plasma flows and turbulence. In particular, the statistical description of transport processes (a new way of thinking in the fusion community) in terms of probability distribution functions replaces the calculation of effective transport coefficients and this is an active area of research in Ciemat.

A probabilistic model of particle transport has been developed, based on the use of probability distributions to describe particle kinetics on the mesoscale, leading to a generalized master equation [12]. The model offers a framework to combine neoclassical and turbulent transport consistently, while clarifying underlying assumptions (e.g. regarding locality) and complex effects (e.g. regarding criticality [13]). A toy model based on these ideas, incorporating a critical gradient mechanism, was shown to exhibit a range of properties very similar to the behaviour of actual fusion transport experiments. Studies of the resulting system show that a straightforward approach based solely on average transport coefficients is probably insufficient to capture the essence of this type of systems, which has important consequences for transport modelling, in general, and transport scaling, in particular.
It has been observed that the ion temperature profile of low-density ECR heated TJ-II plasmas is almost flat and that energetic ions are present well outside the last closed magnetic surface. Ion trajectories with different pitches and starting points have been calculated. The trajectories of ions in TJ-II are computed by considering the actual 3D geometry and the usual drifts due to electric field and the gradient of magnetic field are considered. It has been found that a feasible explanation for such a flat mean energy profile is that ion orbits are wide enough to communicate distant radial parts of the plasma, thus giving an effective flat ion temperature profile for low-density plasmas [14]. Transport analysis performed with the Proctr code shows that the effective diffusivity is pretty high in this flat ion temperature profile (of about $20 \mathrm{~m}^{2} \mathrm{~s}^{-1}$ ), and the CX losses can reach less than $5 \%$ of the power transferred to ions. The typical orbit width has been estimated using a code that estimates ion trajectories in the presence of collisions and electric field in TJ-II and they can reach about $15 \%$ of minor radius [15]. The distribution function is also obtained without considering collisions and non-Maxwellian features are found. The experimental data show that the ion temperature shows a gradient in NBI plasmas in which plasma density and collisionality are higher. In this situation, the electron temperature decreases and approaches the ion value (see figure 4). As both temperatures approach, the energy transmission between both species becomes more efficient, so ion orbits will have less importance for transport and more peaked ion temperature profiles are expected, in accordance with experimental results. 

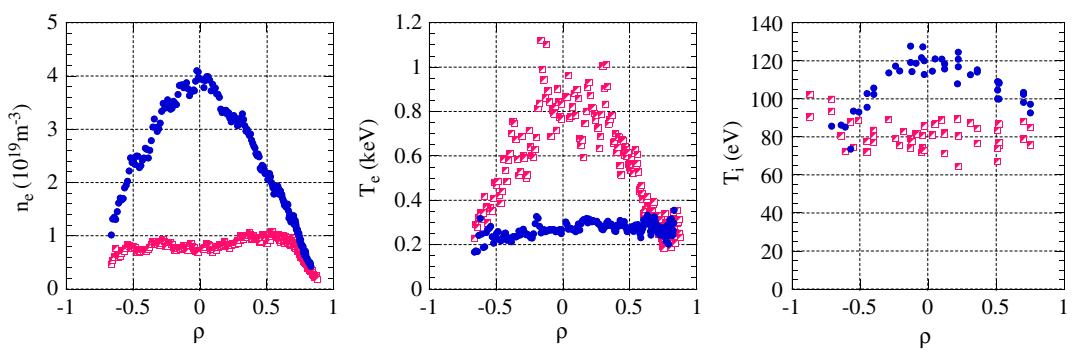

Figure 4. Density and electron and ion temperature profiles in ECH (red open symbol) and NBI (full blue dot) plasmas.

\section{Transitions and magnetic topology}

A transition to improved core electron heat confinement (known as CERC: core electron root confinement) can be triggered when low-order rational magnetic surfaces are located in the core plasma region. Experiments showed a dependence of the threshold density (and the barrier quality) on the order of the rational $(n / m=3 / 2$ versus $4 / 2)$.

The characteristics of CERC triggered by the $n=3 / m=2$ rational surface have already been described in $[16,17]$ and can be summarized as follows. At the transition, the electron temperature increases in the plasma core region $(\rho<0.3)$, while the radial electric field increases substantially - by a factor of three. The HIBP beam current profile indicates that the plasma density profile changes to a slightly more hollow profile during the transition. Quasi-coherent modes are observed as the $E_{r} \times B$ shear flows develop at the CERC formation. The mode can exist before or after the CERC phenomenon but vanishes when the barrier is developed fully [18]. Finally, transitions triggered by the $3 / 2$ rational have no effect on the ion temperature. Recently, a CERC triggered by the $n=4 / m=2$ rational has been studied in TJ-II ECH plasmas. At the barrier formation, an increase in the ion temperature measured by the CX-NPA diagnostic [19] and a reduction in the $\mathrm{H}_{\alpha}$ signals is observed, synchronized with the change in the electron temperature (figure 5). The change in the ion temperature is relatively modest (about 10-15\%), but has not been observed previously either in TJ-II or in other helical devices [20]. As the barrier is lost, ECE traces showed a heat pulse propagating radially outwards. The SXR tomography diagnostic shows a flattening of the profiles localized around $\rho \approx 0.4$ with a $m=2$ poloidal structure (see figure 6). This observation, together with the absence of quasi-coherent oscillations, indicates that the magnetic island associated with the 'natural' resonance $n=4 / m=2$ does not rotate in these discharges. These experiments show that, under some circumstances, the island positioned inside the plasma core region precedes and provides a trigger for CERC formation [21].

\section{Plasma rotation and momentum re-distribution mechanisms}

Comparison of impurity poloidal rotation in ECRH and NBI plasmas. The poloidal rotation of $\mathrm{CV}$ ions has been deduced from spectral line shifts measured using a high spectral resolution spectrometer and a nine fibre-channel system. Figure 7 presents results of $\mathrm{CV}$ poloidal rotation for two
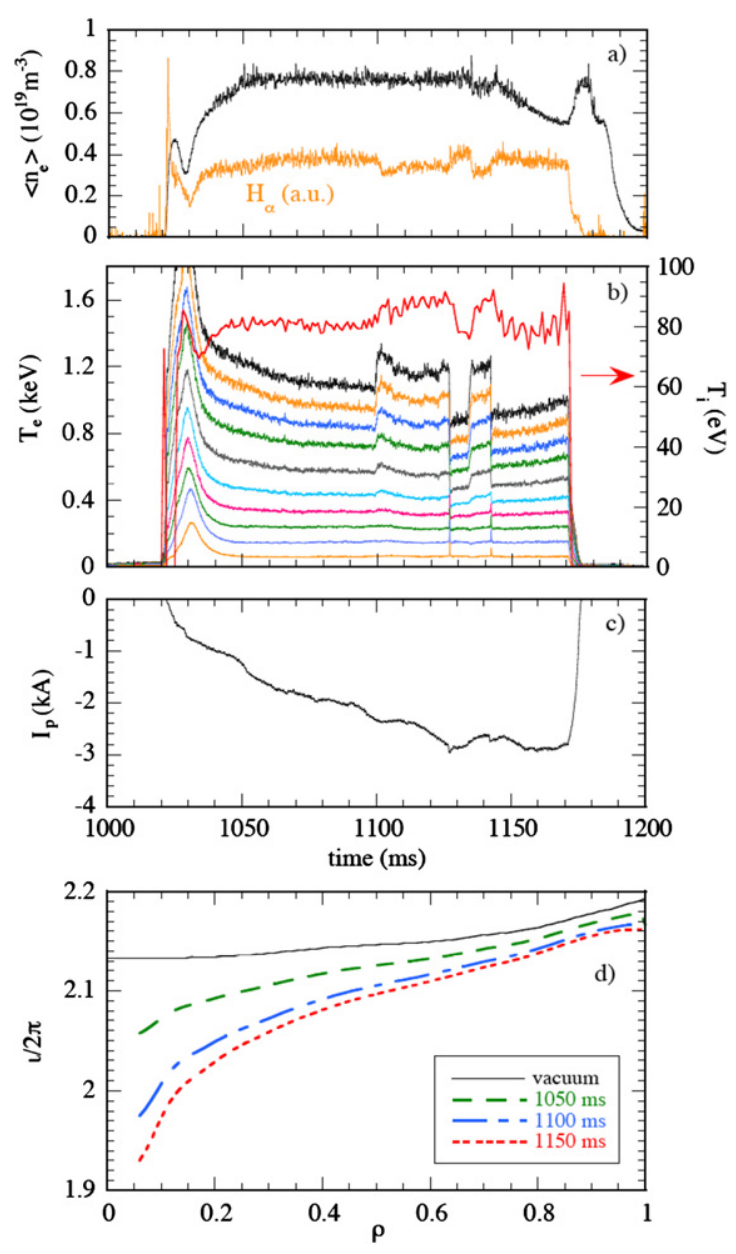

Figure 5. Time evolution of (a) line density and $\mathrm{H}_{\mathrm{a}}$ signal, (b) electron temperature at several radii and ion temperature measured along a central plasma chord, $(c)$ net plasma current and (d) rotational transform in a discharge with $\mathrm{OH}$ induction.

reference ECRH discharges $\left(n_{\mathrm{e}}=0.63\right.$ and $0.67 \times 10^{19} \mathrm{~m}^{-3}-$ open triangles) and for two ECRH+NBI discharges with increasing line-averaged-density $\left(0.94\right.$ and $1.72 \times 10^{19} \mathrm{~m}^{-3}-$ full circles and squares, respectively). The peak $\mathrm{CV}$ rotation corresponding to the upper plasma half is also plotted as a function of the line-averaged electron density. For ECRH plasmas with densities below $0.7 \times 10^{19} \mathrm{~m}^{-3}$ the radial electric field is positive.

Analysis of the data obtained has shown that a change in the sign of the poloidal rotation direction occurs that depends abruptly on plasma density, no matter the heating 

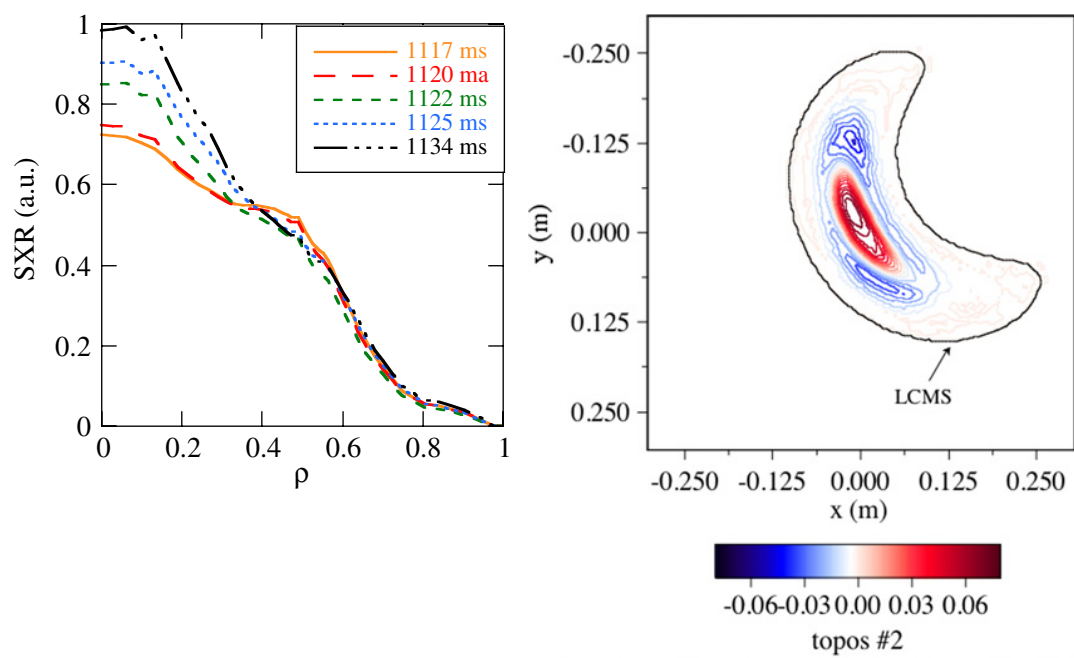

Figure 6. SXR profile evolution during CERC formation (left) and poloidal structure obtained by the SVD analysis of the time evolution of the SXR tomography reconstructions (right). Results show a $m=2$ poloidal structure of the flattening in the SXR profile around $\rho \approx 0.4$.

method. Whereas in low-density plasmas the poloidal direction corresponds to a positive radial electric field, at higher densities negative radial electric fields are deduced from the measured poloidal rotation. These measurements, consistent with HIBP results, are in qualitative agreement with neoclassical theory calculations that predicts that the change in the sign of the radial electric field is mainly due to a change in the ratio of the electron to ion temperature [22].

Properties of spontaneous sheared flows in TJ-II. Experiments showed that the generation of spontaneous perpendicular sheared flows (which self-organize, via fluctuations, to a value close to marginal stability) requires a minimum plasma density. Near this critical density, the level of turbulent edge transport and turbulent kinetic energy increases significantly in the plasma edge [23]. The development of edge sheared flows, first reported by means of Langmuir probe measurements, has been recently visualized in $2 \mathrm{D}$ by means of ultra fast speed cameras, reflectometry and HIBP measurements.

Bright, long-living structures are frequently seen with a spatial extent of few centimetres. These structures, previously referred to as 'blobs', show predominant poloidal movement with typical speeds in the range of $10^{3}-10^{4} \mathrm{~m} \mathrm{~s}^{-1}$, in agreement with the expected ExB drift rotation direction. In addition, the plasma potential measured by HIBP shows a strong dependence on the plasma density. At plasma densities near the threshold value, evidence of inward radial electric fields has been observed at the plasma edge, whereas in the plasma core the radial electric field (as measured by the HIBP system) remains positive. This result implies the simultaneous development of two sheared flows at the threshold density: one located in the proximity of the LCFS $(r / a \approx 1)$ (the one investigated by means of probe measurements and fast cameras) and the other one near $r / a \approx 0.7$. Reflectometry measurements show that the inner shear layer moves inwards as the density increases above the threshold. A waveletbased method was used to characterize turbulent structures geometry and polarity in different poloidal velocity shear regimes [24]. As sheared flows develop, turbulence structures
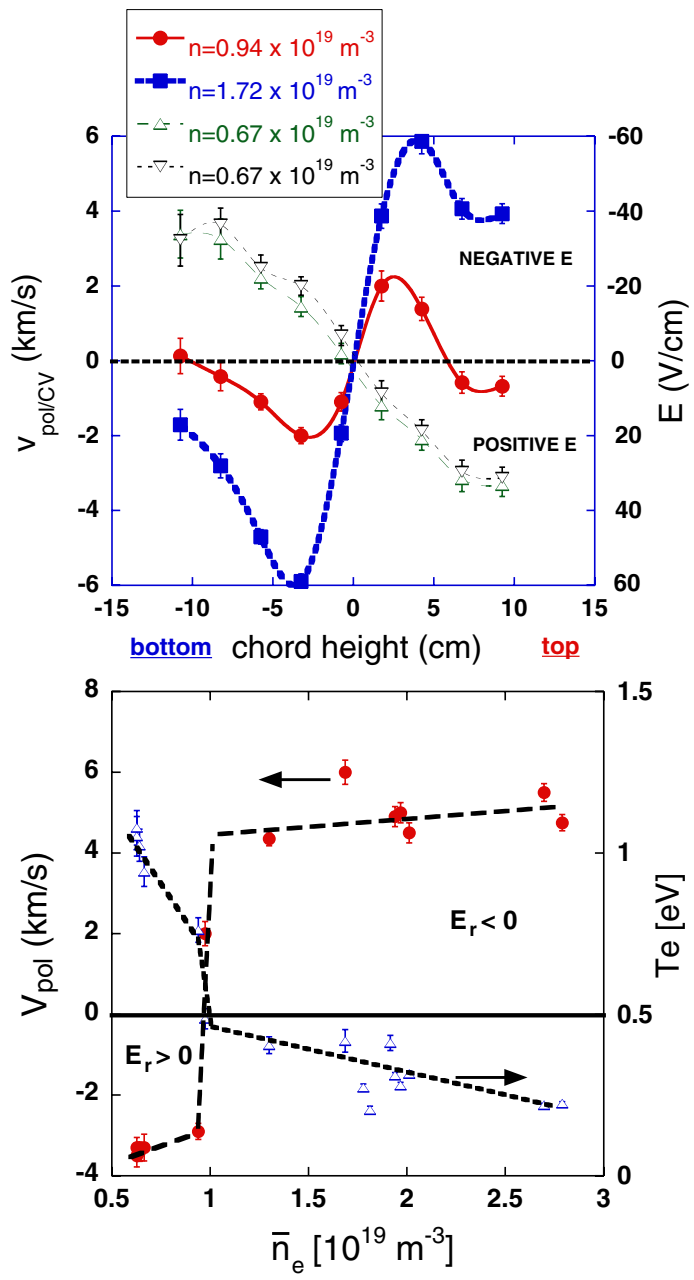

Figure 7. Chord averaged data for $\mathrm{CV}$ poloidal rotation and maximum $\mathrm{CV}$ rotation versus line-averaged density.

become stretched which can be interpreted as a modification in the perpendicular degree of turbulence anisotropy (figure 8). This result is consistent with a basic prediction of the shear decorrelation model (put forward more than 15 years ago). 


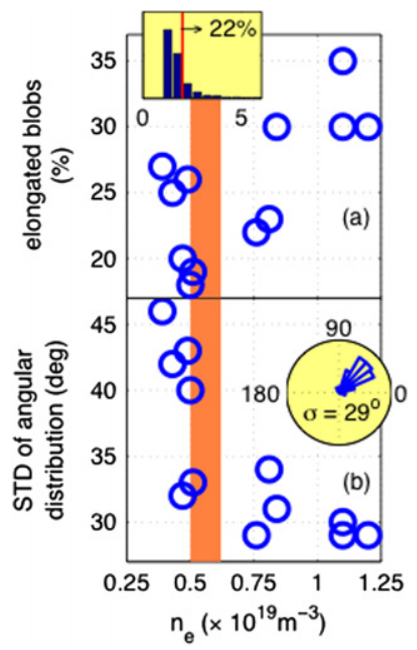

Figure 8. During the development of spontaneous edge sheared flows, fluctuations in the angular distribution of blobs are reduced and the elongation of blobs increases.

Recently, it has been shown that experimental results concerning the emergence of the plasma edge shear flow layer in TJ-II can be explained using a simple model for a second order transition based on shear flow amplification by Reynolds stress and turbulence suppression by shearing. In the dynamics of the model, the resistive interchange instability was used (figure 9). This model predicts a power dependence on density gradients before and after the transition, consistent with experiment [25]. In the framework of this interpretation, the observed spontaneous transitions to improved confinement [26], triggered by edge sheared flows which organize themselves near marginal stability, could be interpreted as the first step (second order phase transition) leading to an $\mathrm{H}$ mode transition.

Finally combined ECRH and NBI experiments reveal that, once ECRH heating power is turned off, a confinement regime characterized by a strong reduction in $E \times B$ turbulent transport, an increase in the perpendicular velocity (by about a factor of 2) and a significant increase in plasma density is achieved. Whether this transition can be related to a fully developed $\mathrm{H}$ mode transition, in which perpendicular flows are dominated by pressure gradients, still remains an open question.

Relaxation of electric fields during electrode biasing experiments. The effect of electrode biasing on plasma confinement, turbulence and plasma flows has been studied. Experimental results show that it is possible to modify global confinement and edge plasma parameters with biasing, and evidence of electric field-induced improved confinement has been found. The plasma response is different at densities below and above the threshold value needed to trigger the spontaneous development of $E \times B$ sheared flows in TJ-II.

Different driving and damping mechanisms (neoclassical and anomalous) have been proposed to explain the development of radial electric fields and plasma flows in the plasma boundary region of fusion devices. To shed some light on this problem, the decay times of edge potential and perpendicular flows measured in the edge plasma region have been investigated when the electrode applied potential is turned off.

The time evolution of floating potential signals have been fitted to an exponential function, $U_{\mathrm{fl}}(t)=U_{\max } \exp (-t / \tau)+$ $U_{\min }$ where $\tau$ is the relaxation time. This fitting procedure has been applied to floating potential signals measured at the plasma edge of the TJ-II by Langmuir probes, at different densities and different magnetic configurations (figure 10). Two time scales have been found for the decay of the edge plasma potential, measured when the applied electrode potential is turned off. On the fast time scale $(10-50 \mu \mathrm{s})$ the plasma potential changes by about $50-100 \mathrm{~V}$ after biasing in the edge region; on the slow time scale (comparable to the particle confinement time) plasma potential modifications are linked to the evolution of the plasma density. No clear relation between the fast decay time and plasma density has been found so far, but results might suggest an increase in decay times above the threshold density value. These results can shed some light on neoclassical and anomalous damping mechanisms in fusion plasmas.

Energy transfer between flows and turbulence. Experimental results also show significant parallel turbulent forces (proportional to quadratic terms in fluctuating velocities) at plasma densities above the threshold value to trigger perpendicular $E \times B$ sheared flows [27].

Recently, a new method has been applied for the quantification of the local energy transfer between flows and turbulence, by computing the production term $(P)[27,28]$. The radial-parallel contribution to the turbulence production term is proportional to the radial derivative of the mean parallel velocity and the radial-parallel component of Reynolds stress (figure 11).

Experimental results show that turbulence can act both as an energy sink and an energy source for the mean flow near the shear layer. These parallel turbulent forces are mainly localized at the radial location where sheared flows develop. Present findings are consistent with numerical simulations, emphasizing the role of turbulent forces on both perpendicular and parallel flow components during the development of zonal flows [29]. TJ-II results show that shear flow physics involves 3D physics phenomena in which both perpendicular and parallel dynamics play a role.

\section{MHD studies}

Previous experiments have shown an increase in fluctuation levels in configurations with magnetic hill in the plasma edge region. However, although the magnetic well is the main stabilizing mechanism in TJ-II, plasma profiles are not dramatically affected when the magnetic well is removed in the plasma edge. This result, consistent with recent findings in the LHD stellarator [30], calls into question stability calculations based on the assumption of smooth pressure profiles.

ELM-like events have been reported in TJ-II magnetic configurations with rational magnetic surfaces $(3 / 2,5 / 3,8 / 5)$ in certain plasma parameters windows (e.g. high density). An analysis of ELM-like events, based on the coupling of iontemperature-gradient modes to Alfvén and acoustic modes, has been made [31]. 

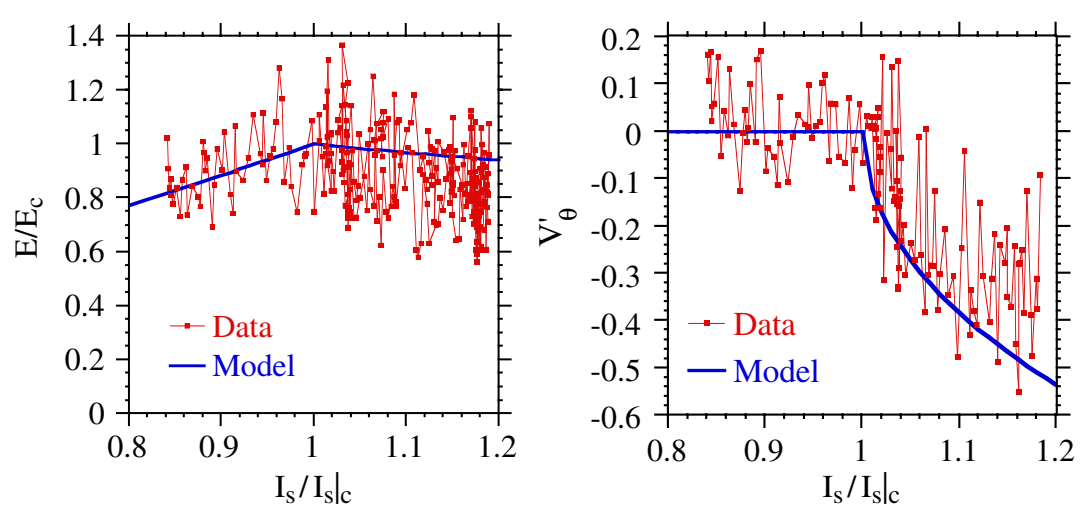

Figure 9. Evolution of the level of turbulence $(E)$ and the averaged poloidal velocity shear versus the normalized averaged ion saturation current from the model, in which the basic mechanism for the emergence of the shear flow is the Reynolds stress, and experimental results in the edge of the TJ-II stellarator.
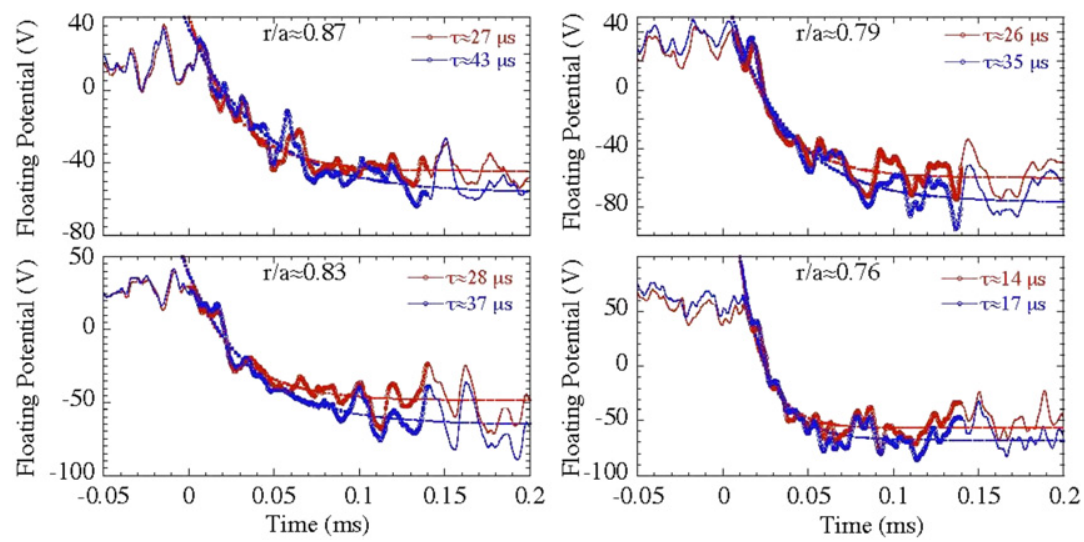

Figure 10. A fit of the floating potentials decay measured at different radial probe positions in TJ-II. Biasing was switch off at time $=0$. The decay time, in the range $10-40 \mu \mathrm{s}$, decreases in the plasma edge region.

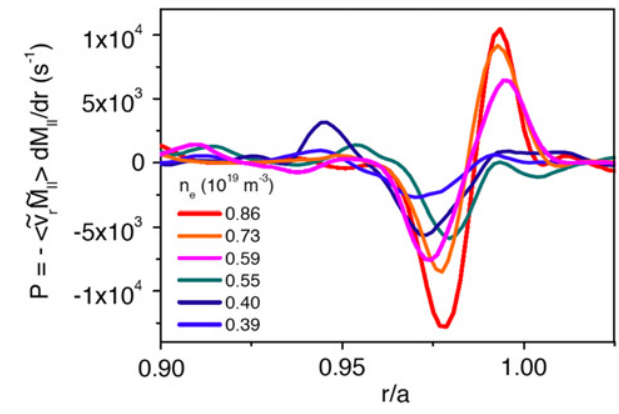

Figure 11. Parallel-radial component of the turbulence production term during edge sheared flow development in the TJ-II stellarator. Evidence of significant energy transfer between turbulence and parallel flows at the on-set of sheared flows was found.

Magneto-hydro-dynamic instabilities in the TJ-II stellarator are being characterized experimentally in various plasma parameter regimes and heating scenarios [32]. The appearance of low frequency modes (some tens of $\mathrm{kHz}$ ) in $\mathrm{ECH}$ plasmas depends on the rotational transform profile and the plasma density. In NBI plasmas, high frequency $(150-300 \mathrm{kHz})$ modes have been found in plasmas with line densities in the range $0.6-$ $3 \times 10^{19} \mathrm{~m}^{-3}$ and heated with on/off-axis electron cyclotron heating. They are likely global Alfven eigenmodes (GAEs) related to the low order resonance. These high frequency modes are found in different configurations and related to several low-order rationals, $n / m=3 / 2,5 / 3$. The Alfvén modes appear as coherent and continuous oscillations whose frequency shows a clear dependence with plasma density $\left(f \approx 1 / n^{0.5}\right)$ (figure 12 ).

\section{Plasma-wall studies}

Plasma-wall studies at Ciemat encompass reactor-oriented work [33] as well as TJ-II experiments. In what follows, only the latter type of activities are described.

Deposition and erosion of carbon films from hydrocarbon puffing in TJ-II. Following previous studies on impurity screening in TJ-II, comparative studies of the injection of methane $\left(\mathrm{CH}_{4}\right)$, ethylene $\left(\mathrm{C}_{2} \mathrm{H}_{4}\right)$ and hydrogen $\left(\mathrm{H}_{2}\right)$ through a mobile, instrumented graphite limiter were investigated [33]. Gas pulses of $12-15 \mathrm{~ms}$, corresponding to the injection of $(4-8) \times 10^{18}$ particles, were produced at a fixed time during the discharge. The plasma density, local $\mathrm{H}_{\alpha}$ emission and central carbon density $(\mathrm{CV})$ showed a more or less significant increase as the species were injected, whereas no significant changes in the edge electron temperature were recorded by the $\mathrm{He}$ line ratio method. When comparing $\mathrm{C}_{2} \mathrm{H}_{4}$ and $\mathrm{H}_{2}$ injection, a much lower fuelling efficiency was deduced for ethylene, in spite of its higher electron/molecule content. This is also 


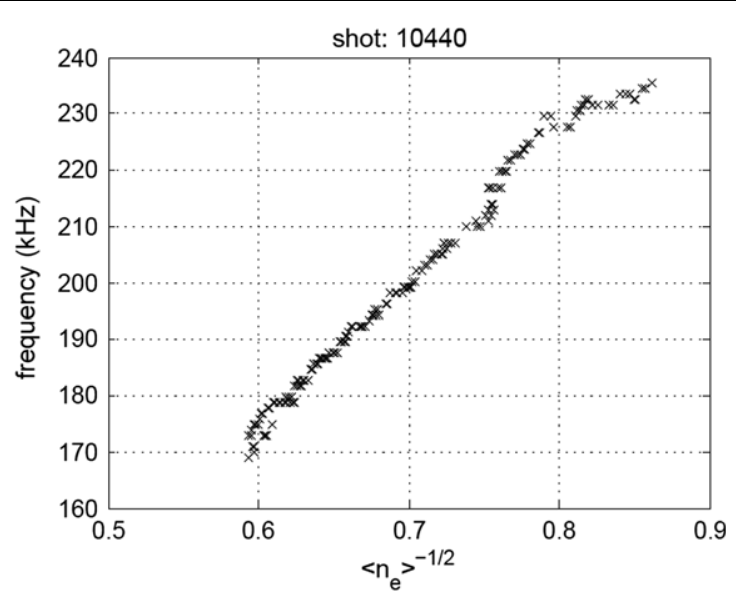

Figure 12. Frequency of plasma modes (as measured by Mirnov coils) versus density in NBI plasmas.

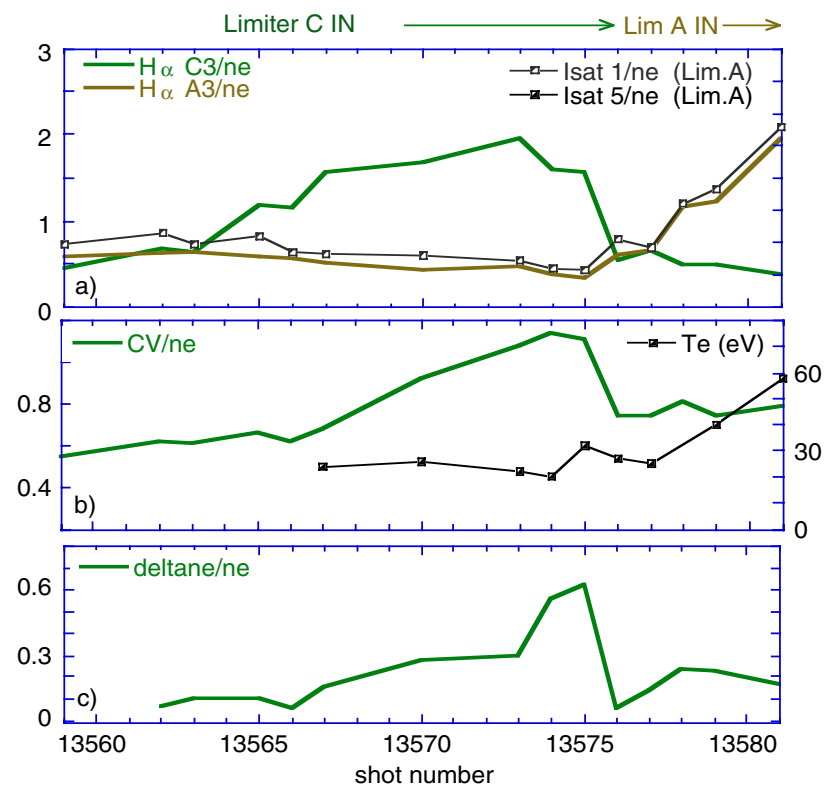

Figure 13. Effects of limiter insertion in the plasma depending on hydrocarbon deposition. Limiter C, contaminated. Limiter A, clean.

true for the increase in local $\mathrm{H}_{\alpha}$ emission, regardless of the higher $\mathrm{H} /$ molecule ratio of $\mathrm{C}_{2} \mathrm{H}_{4}$. For this species, the relative $\mathrm{H}_{\alpha}$ photon yield per injected $\mathrm{H}$ atom is only $15 \%$ of that of hydrogen. If $\mathrm{CH}_{4}$ and $\mathrm{C}_{2} \mathrm{H}_{4}$ are compared with respect to their spectroscopic signatures, it is found that the ratio of $\mathrm{CH} / \mathrm{H}_{\alpha}$ emission intensities is a factor of 3 higher for the case of $\mathrm{C}_{2} \mathrm{H}_{4}$ at the typical edge conditions of the plasma, $T_{\mathrm{e}}=30 \mathrm{eV}$ and $n_{\mathrm{e}}=2 \times 10^{12} \mathrm{~cm}^{-3}$. All this information can be understood from the point of view of the prompt deposition of carbon layers with high $\mathrm{H}$ content when ethylene is fed into the plasma.

The topic of local contamination by the injection of hydrocarbons is specifically addressed in the experiments of limiter insertion shown in figure 13. Ethylene was injected using $15 \mathrm{~ms}$ pulses during a radial scan of the limiter position. In each scan, the symmetric limiter was kept at the location of the nominal LCFS. Thus, limiter $\mathrm{C}$ (the one that carries the gas inlet) was gradually inserted from the normalized minor radius $r=1$ up to $25 \mathrm{~mm}$ inside the LCFS $(r \sim 0.8)$ in discharges $<13575$. From 13576 to 13582 , the reverse was done, i.e. limiter $\mathrm{A}$ was inserted while limiter $\mathrm{C}$ remained at the LCFS position. Data shown in parts (a) and (b) of the figure refer to the parameter value before the pulse is produced $(t<1120 \mathrm{~ms})$. As seen in the figures, a local enhancement of recycling is observed only at the inserted limiter. It must be kept in mind that the total displacement of the limiter is more than twice the corresponding density decay length of the SOL $(\sim 1 \mathrm{~cm})$ of this type of plasmas. As ethylene is puffed during the whole scan, a local amorphous carbon layer may be created near the injection point. Evidence of this is shown by the systematic increase in the plasma carbon content as limiter $\mathrm{C}$ is inserted shot by shot (figure 13(b)). Conversely, a constant value (although higher than the initial one) of this plasma impurity is found when the carbon limiter A is moved inside the LCFS, thus indicating that simple erosion of the graphite limiter is not responsible for the enhancement. Figure 13(c) shows the fuelling effect of the injected ethylene. It is seen that a fairly constant value is reached for all limiter settings and only for the innermost locations of the limiter ( $>2 \mathrm{~cm}$ in) does an enhanced effect take place. This value, however, represents only $2 \%$ of the available electrons in the pulse.

The formation and erosion of films created by hydrocarbon injection was studied in a new experiment. First, a single pulse of the hydrocarbon species $\left((2-4) \times 10^{18}\right.$ molec $)$ was injected through the limiter into the plasma. Then, the gas was replaced by hydrogen and a series of repetitive pulses were made. The process was repeated at several positions of the limiter and for both hydrocarbons. Alternatively, hydrogen was fed into the plasma through a valve far from the observation region, so that no extra neutral density increase, aside from that expected from local recycling, was foreseen. The ratio of enhanced $\mathrm{CH}$ emission to that of local $\mathrm{H}_{\alpha}$ was found to be independent of the type of molecule used as a precursor of the film depending on hydrocarbon deposition. Secondly, and contrary to simple expectations, a higher erosion efficiency by plasmas fed away from the limiter is also observed. As physical sputtering is the major contribution to carbon contamination in TJ-II [34], this result seems to be due to the contribution of other contaminated parts of the vessel. As a consequence, the expected increase in chemical erosion by enhancing the atomic $\mathrm{H}$ density near the injection port could not be detected.

EIRENE simulation of Helium plasmas. The EIRENE Montecarlo code has been applied for the first time to the simulation of a He plasma produced in the TJ-II stellarator by ECRH [35]. Edge $n_{\mathrm{e}}$ and $T_{\mathrm{e}}$ profiles have been deduced by the supersonic $\mathrm{He}$ beam, but obtained in similar $\mathrm{H}$ plasmas. A search for the Eirene parameters that produce agreement between the experimental data for chord integrated He emission lines at three different wavelengths was carried out. The most sensitive parameters correspond to the shape of the radial profiles near the plasma periphery. Optimal values, well within the experimental error limits and the Monte Carlo statistical uncertainty, have been found (maximum difference $15 \%$, average $9 \%$ ), and the corresponding neutral profiles have been obtained and analysed. Absolute calibrated emissivity values, as well as signal ratios, have been used in the fits. However, the present simulation cannot solve uncertainties on the ratio of the edge/central neutral density and the concomitant uncertainty in the kinetic energy of the recycling neutrals. 
Neutral hydrogen profile modelling in TJ-II. The profiles of the neutral molecular hydrogen, $\mathrm{H}_{2}$, and that of atomic hydrogen, $\mathrm{H}$, in front of a poloidal limiter of TJ-II have been modelled using a simple 1D model and the EIRENE Monte Carlo Code [30] and compared with $\mathrm{H}_{\alpha}$ plasma imaging with a CCD camera. Very similar profiles were obtained with both methods if the product energy of the hydrogen atoms after the molecular dissociation is taken to be $\sim 0.3 \mathrm{eV}$. For ECRH plasmas, the screening of neutrals by the plasma is rather low in TJ-II, and the neutral penetration to the core plasma can be responsible for high CX losses of hot ions when NBI is switched on.

Lithiumization of TJ-II. In order to explore, for the first time in a stellarator, the plasma behaviour under a low $Z$, low recycling scenario, full coating of the inner walls of TJ-II by $\mathrm{Li}$ was done. This was achieved by feeding a Ne GD plasma with lithium vapour from a set of four effusive ovens, symmetrically located in TJ-II. A total of $4 \mathrm{~g}$ of Li were injected, which should provide good density control for ECRH and NBI plasmas in the next campaign.

\section{Conclusions}

- The investigation of plasma potential profiles reveals a direct link between electric fields, density and plasma confinement. The smooth change from a positive to a negative electric field observed in the core region as the density is raised is correlated with global and local transport results, showing a confinement time improvement and a reduction of electron transport. The statistical description of transport is emerging as a new way to describe the coupling between profiles, plasma flows and turbulence.

- TJ-II experiments show that the location of rational surfaces inside the plasma can, in some circumstances, provide a trigger for development of core transitions. TJ-II findings provide a critical test for different models proposed to explain the appearance of ITBs linked to magnetic topology.

- The investigation of momentum transport mechanisms in the core and edge region is emerging as a key area of research in the TJ-II stellarator. In the plasma core, perpendicular rotation is strongly coupled to plasma density, showing a reversal consistent with neoclassical expectations. In contrast, spontaneous sheared flows appear to be strongly coupled to plasma turbulence in the plasma edge, consistent with expectations for turbulencedriven flows.

- The local injection of hydrocarbons through a mobile limiter and the erosion by plasmas with well-known edge parameters open the possibility of carbon transport studies relevant to co-deposit formation in fusion devices. The erosion pattern of the resulting hydrocarbon films was studied and compared with that of bulk graphite.

\section{References}

[1] Hidalgo A. et al 2006 Plasma Phys. Control. Fusion 48527

[2] Carmona J.M. et al 2006 Rev. Sci. Instrum. 77 10F107

[3] Sanchez M. et al 2005 Rev. Sci. Instrum. 76046104

[4] Rapisarda D. et al 2006 Rev. Sci. Instrum. 77033506

[5] Yamada H. et al 2005 Nucl. Fusion 45 1684-93

[6] Vargas V.I. et al 2007 Nucl. Fusion submitted

[7] Fujisawa A. et al 2003 Plasma Phys. Control. Fusion 45 R1

[8] Medina F. et al 2005 Proc. 32nd EPS Conf. on Controlled Fusion and Plasma Physics (Tarragona, Spain) P5-026

[9] Ochando M. et al 2006 Fusion Sci. Technol. 50313

[10] Guasp J. and Liniers M. 2000 Nucl. Fusion 40411

[11] Ochando M. et al 2006 Plasma Phys. Control. Fusion 481573

[12] Milligen B. Ph. 2005 Plasma Phys. Control. Fusion 47 B743

[13] Carreras B.A. et al 2006 Phys. Plasmas 13062301

[14] Castejón F. et al 2006 Fusion Sci. Technol. 50412

[15] Castejón F. et al 2007 Plasma Phys. Control. Fusion submitted

[16] Estrada T. et al 2004 Plasma Phys. Control. Fusion 46277

[17] Castejón F. et al 2004 Nucl. Fusion 44593

[18] Estrada T. et al 2005 Plasma Phys. Control. Fusion 47 L57-L63

[19] Fontdecaba J.M. et al 2004 Fusion Sci. Technol. 46271

[20] Yokoyama M. et al 2006 Fusion Sci. Technol. 50327

[21] Estrada T. et al 2007 Nucl. Fusion 47305

[22] Zurro B. et al 2006 Fusion Sci. Technol. 50419

[23] Pedrosa M.A. et al 2005 Plasma Phys. Control. Fusion 47777

[24] Alonso A. et al 2006 Plasma Phys. Control. Fusion 48 B465

[25] Carreras B.A. et al 2006 Plasma Phys. 13 122509-1

[26] Tabares F.L. et al 2001 Plasma Phys. Control. Fusion 431023

[27] Gonçalves B. et al 2006 Phys. Rev. Lett. 96145001

[28] Sanchez E. et al 2005 J. Nucl. Mater. 337296

[29] Hallatschek K. 2004 Phys. Rev. Lett. 93065001

[30] Watanabe K.Y. et al 2005 Nucl. Fusion 451247

[31] Jimenez J A et al 2006 Plasma Phys. Control. Fusion 48515

[32] Jimenez R. et al 2006 Plasma Phys. Control. Fusion 48515

[33] Tafalla D. et al 2006 Proc.Conf. on Plasma Surface Interactions (Hefei, China)

[34] Garcia-Cortes I. et al 2005 J. Nucl. Mater. 337-339 441

[35] Tabares F.L. et al 2006 Proc. 33rd EPS Conf. on Plasma Physics (Frascati, Italy) 